\title{
Qualidade microbiológica, sensorial e físico-química de geleia de abacaxi com diferentes concentrações de pimenta
}

\author{
Microbiological, sensorial and physical-chemical quality of pineapple jelly with different \\ concentrations of pepper \\ Calidad microbiológica, sensorial y físico-química de la jalea de piña con diferentes \\ concentraciones de pimienta
}

\section{Resumo}

A geleia é um tipo de doce que pode ser produzida com açúcar ácido e pectina. Com a finalidade de aproveitamento dos frutos de pimenta e abacaxi, cultivados em todo o território nacional, representativamente na agricultura familiar, é necessário o desenvolvimento de produtos que atendam às necessidades dos consumidores. O objetivo do trabalho foi avaliar a qualidade e a preferência de geleia de pimenta de bode (Capsicum chinenses L.) sob diferentes concentrações. Para a fabricação da geleia de pimenta, os frutos foram colhidos no setor de horticultura do IF Goiano - Campus Ceres e passaram pelo processo de sanitização em água clorada. Após esse processo foi realizada a fabricação da geleia de pimenta com abacaxi. Foram realizadas análises físico-químicas e microbiológicas no laboratório instrumental e microbiológico, verificando o nível qualitativo e de contaminação da geleia. Em seguida foi realizada a análise sensorial com 50 provadores não treinados, a fim de avaliar o índice de aceitabilidade. Os tratamentos foram (T1-0,2, T2-0,4, T30,6, T4-0,8) gramas de pimenta de bode, em cada geleia produzida. Os dados foram submetidos ao teste de Tukey a $5 \%$ de probabilidade, na planilha eletrônica da UFSCar. As amostras avaliadas apresentaram o número de NMP $\leq 3$, estando dentro dos padrões microbiológicos. Os resultados das análises físico-químicas demostram que a geleia está dentro dos padrões da literatura. A análise sensorial apresentou o índice de aceitabilidade superior $70 \%$ apresentando valores entre $80 \%$ e $90 \%$. Os tratamentos (T1-0,2; T4-0,8) com menor e maior concentração de pimenta foram os que tiveram melhor preferência.

Palavras-chave: Capsaicina; Comercialização; Higiene; Sabor.

\begin{abstract}
Jelly is a type of candy that can be made with sour sugar and pectin. In order to use pepper and pineapple fruits, cultivated throughout the national territory, representatively in family farming, it is necessary to develop products that meet the needs of consumers. The objective of this work was to evaluate the quality and preference of goat pepper jelly (Capsicum chinenses L.) under different concentrations. For the manufacture of pepper jelly, the fruits were collected in the horticulture sector of the IF Goiano - Campus Ceres and went through the sanitization process in chlorinated water. After this process, pepper jelly with pineapple was manufactured. Physical-chemical and microbiological analyzes were performed in the instrumental and microbiological laboratory, checking the qualitative level and contamination of the
\end{abstract}


jelly. Then, a sensory analysis was performed with 50 untrained tasters, in order to assess the acceptability index. The treatments were (T1-0.2, T2-0.4, T3-0.6, T4-0.8) grams of goat pepper in each jelly produced. Data were submitted to the Tukey test at $5 \%$ probability, in the UFSCar electronic spreadsheet. The evaluated samples presented the number of $\mathrm{NMP} \leq 3$, being within the microbiological standards. The results of the physicochemical analyzes show that the jelly is within the standards of the literature. The sensory analysis showed an acceptability index higher than $70 \%$, with values between $80 \%$ and $90 \%$. The treatments (T1-0.2; T4-0.8) with the lowest and highest concentration of peppers were the ones that had the best preference.

Keywords: Capsaicin; Commercialization; Flavor; Hygiene.

\section{Resumen}

La jalea es un tipo de dulce que puede producirse con azúcar ácido y pectina. Con el fin de utilizar los frutos de la pimienta y la piña, cultivados en todo el territorio nacional, representativamente en la agricultura familiar, es necesario desarrollar productos que satisfagan las necesidades de los consumidores. El objetivo del trabajo fue evaluar la calidad y la preferencia de la jalea de pimiento de cabra (Capsicum chinenses L.) bajo diferentes concentraciones. Para la fabricación de la jalea de pimiento, los frutos se cosecharon en el sector hortícola del IF Goiano - Campus Ceres y se sometieron al proceso de higienización en agua clorada. Después de este proceso se realizó la fabricación de la jalea de pimienta con piña. Se realizaron análisis fisicoquímicos y microbiológicos en el laboratorio instrumental y microbiológico, comprobando el nivel cualitativo y la contaminación de la jalea. A continuación, se realizó un análisis sensorial con 50 catadores no entrenados para evaluar el índice de aceptabilidad. Los tratamientos fueron (T1-0,2, T20,4, T3-0,6, T4-0,8) gramos de pimiento de cabra en cada jalea producida. Los datos se sometieron a la prueba de Tukey al 5\% de probabilidad, en la hoja de cálculo electrónica de UFSCar. Las muestras evaluadas presentaron el número de $\mathrm{MPN} \leq 3$, estando dentro de los estándares microbiológicos. Los resultados del análisis físicoquímico demuestran que la jalea está dentro de los estándares de la literatura. El análisis sensorial presentó el índice de aceptabilidad superior al $70 \%$ presentando valores entre el $80 \%$ y el $90 \%$. Los tratamientos (T1-0,2; T4-0,8) con menor y mayor concentración de pimienta fueron los de mayor preferencia.

Palabras clave: Capsaicina; Comercialización; Higiene; Sabor.

\section{Introdução}

O abacaxi (Ananas comosus L.), é uma espécie tropical de fruto pertencente à família Bromeliaceae, que é altamente apreciado por seu sabor característico, aroma e propriedades nutricionais (Silva et al., 2019). A composição química do abacaxi varia muito de acordo com a época em que é produzido, gerando frutos com maior teor de açúcar e menor acidez no verão (Pereira et al., 2015). A água é o principal componente do abacaxi, constituindo cerca de 80-85\% do fruto, aumentando sua perecibilidade (Shaari et al., 2018). Devido a curta vida de prateleira do abacaxi, se fazem necessárias pesquisas que busquem alternativas de processamento que possam reduzir essas perdas econômicas (Raghuwanshi et al., 2019; Silva et al., 2019).

O cultivo de pimenta no país tem grande importância devido aos seus benefícios como, boa rentabilidade, agregação de valor ao produto e pela importância social por empregar elevado número de mão de obra, especialmente na época da colheita e processamento (Cruz et al., 2019). De acordo com Macedo et al. (2017), o cultivo da pimenta é de grande importância pela sua rentabilidade, principalmente quando é agregado valor ao produto como; conservas, molhos, geleias, páprica, devido ao elevado uso de mão-de-obra, tem grande importância social.

Devido ao seu sabor, as pimentas constituem um grupo muito peculiar podem apresentar sabor doce ou picante outra característica que a torna peculiar é a estimulação as funções digestivas, sendo parte da dieta de um quarto da população do planeta (Barduzzi, 2011). As pimentas possuem um alto valor nutricional, isso devido à substância presente no fruto chamada de capsaicina responsável pela pungência. Essa sustância é rica em vitamina $\mathrm{A}, \mathrm{B}_{1}, \mathrm{~B}_{2}, \mathrm{C}$, E, e PP. Além de todas as vitaminas presentes, também possui propriedades analgésicas e energéticas, reduz coágulos no sangue (devido à função vasodilatadora), estimula a produção de endorfina e é antioxidante (Melo \& Costa, 2011).

A produção industrial e artesanal de geleias tem como uma das características principais agregar valor as matériasprimas, tanto de grandes como de pequenos produtores, assim amenizando os problemas de falta de fornecimento para os consumidores. Os frutos de abacaxi, principalmente aqueles com menor valor de prateleira para consumo in natura e menor tempo de vida útil, podem ser processados na forma de geleias de forma a evitar perdas e permitir seu consumo por períodos 
prolongados (Shinwari \& Rao, 2018). A geleia é definida como produto obtido pela cocção de frutas inteiras ou em pedaços, ou da polpa ou do suco de frutas, com açúcar e água e concentrado até atingir uma consistência gelatinosa (Brasil, 1978; Pereira et al., 2015). As geleias surgiram com o intuito de disponibilizar as frutas durante a entressafra, como produtos estáveis nas prateleiras, além disso, é uma alternativa para minimizar o desperdício causado pela perecibilidade das frutas (Rodrigues et al., 2020).

O objetivo deste trabalho foi avaliar a qualidade microbiológica, sensorial e físico-química de geleia de abacaxi enriquecida com diferentes concentrações de pimenta bode (Capsicum chinenses L.).

\section{Metodologia}

A elaboração da geleia de abacaxi com pimenta foi realizada no setor de Agroindústria do Instituto Federal Goiano Campus Ceres. As análises foram realizadas no Laboratório Instrumental e microbiológico do Instituto Federal Goiano - Campus Ceres. Foram utilizados frutos de pimentas de bode (Capsicum chinenses L.) provenientes do setor de horticultura do Instituto Federal Goiano-Campus Ceres. O açúcar, pectina e os frutos de abacaxi utilizados como matéria-prima para a elaboração desse projeto foram adquiridos no comércio local da cidade de Ceres-GO.

Seguindo a metodologia de Souza (2017), no processo de preparação da geleia, foi cortado $250 \mathrm{~g}$ de abacaxi e o mesmo foi triturado no liquidificador contendo $250 \mathrm{~mL}$ de água. A polpa resultante do processamento do abacaxi foi colocada em uma panela. Após foi adicionado $250 \mathrm{~mL}$ de água para cada concentração de pimenta constituindo os tratamentos (T1-0,2; T2- 0,4; T2-0,6 e T-0,8) gramas de pimenta de bode, no liquidificador para o processo de trituração. Após esse procedimento foi realizado a mistura da polpa do abacaxi com a polpa de pimenta e em seguida foi adicionado $250 \mathrm{~g}$ de açúcar e levado ao fogo baixo até o ponto de fervura, onde foi adicionado $250 \mathrm{~g}$ de açúcar e $5 \mathrm{~g}$ de pectina comercial (MAGO). Foi misturada até dar consistência, formando um fio ao levantar a colher. Como conservante foi utilizado uma colher de sopa com $2 \mathrm{~mL}$ de caldo de limão. A geleia foi envazada em vidrarias com capacidade de $350 \mathrm{~mL}$. Para selar a tampa e esterilizar o produto as vidrarias foram viradas para baixo por um período de dois minutos.

Foram realizadas análises microbiológicas no laboratório de microbiologia do Instituto Federal Goiano-Campus Ceres, para a determinação de contaminação por coliformes totais, coliformes termotolerantes, pela técnica de tubos múltiplos e o resultado expresso em NMP/g de acordo com as técnicas descritas por Silva et al. (1997). Esta análise foi realiza a fim de constatação da qualidade e a segurança de consumo do produto, quanto a possíveis contaminações por microrganismos.

Os testes sensoriais de aceitação por atributos (textura, aroma, cor, sabor, aspectos gerais) das amostras de geleia foram avaliados por meio de teste afetivo, em local central, conduzidos com a participação de 50 provadores não treinados. Participaram do teste alunos e servidores do Instituto Federal Goiano - Campus Ceres, de ambos os sexos e maiores de 18 anos. Os provadores avaliaram as amostras, mediante escala hedônica estruturada de nove pontos (Figura 1), variando de 1 (desgostei muitíssimo) a 9 (gostei muitíssimo), segundo Minim (2010). As aceitações das amostras foram avaliadas de forma livre com cerca de $25 \mathrm{~g}$ de cada amostra, sendo servida em copos descartáveis de $50 \mathrm{~mL}$ acompanhado com bolacha água e sal. 
Figura 1. Ficha de análise sensorial.

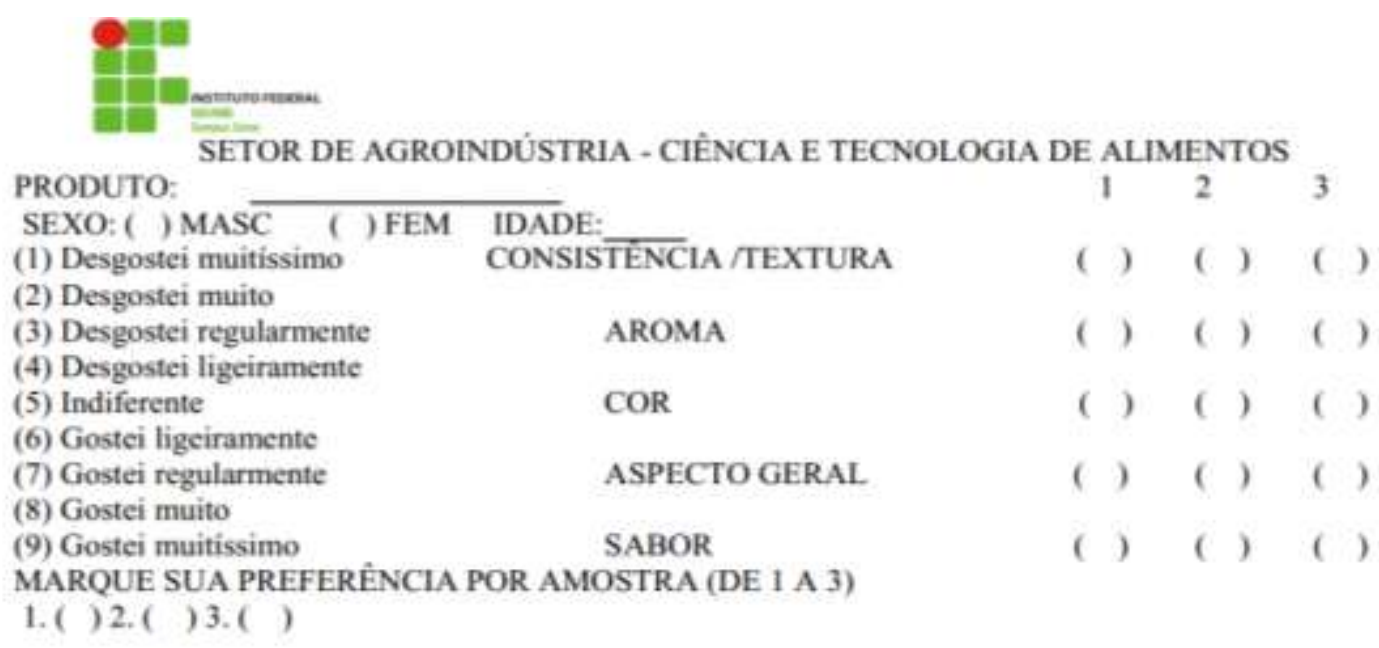

Fonte: Arquivo Pessoal (2018).

A geleia de abacaxi com diferentes concentrações de pimenta foi avaliada quanto ao $\mathrm{pH}$, acidez titulável, umidade e cinzas, no laboratório Instrumental do Instituto Federal Goiano - Campus Ceres. As análises foram realizadas de acordo com as metodologias do Manual de Métodos Físico-Químicos para Análise de Alimentos, do Instituto Adolfo Lutz (2005), em que cada avaliação foi realizada em triplicata.

Os resultados dos testes sensoriais de aceitação, por atributos, foram submetidos à análise de variância e as diferenças das médias comparadas pelo teste de Tukey ao nível de 5\% de significância. Os resultados do índice de aceitabilidade foram avaliados em porcentagem. Para a análise estatística dos testes físico-químicos e microbiológicos, foi utilizado o delineamento experimental inteiramente casualizado utilizando-se o software SISVAR 5.6, Ferreira (2014).

\section{Resultados e Discussão}

De acordo com os resultados obtidos na análise microbiológica (Tabela 1), todos os tratamentos apresentaram < 3,0 NMP/g, exceto T4, que apresentou $\leq 3,0$ NMP/g. De acordo o Anexo I - Padrões Microbiológicos Sanitários para Alimentos da Resolução-RDC No 12, de 02 de janeiro de 2001, a tolerância para amostra é de 5 NMP/g para geleias, mostrando que a amostra T4 (0,8 g de pimenta) mesmo com contaminação na diluição $10^{-2}$, permaneceu dentro dos parâmetros legais estabelecidos. As demais amostras não apresentaram nenhuma contaminação assim tendo <3,0 NMP/g.

Tabela 1. Resultados das análises microbiológica de coliformes totais (CTO) e coliformes termotolerantes (CTT) de geleia de abacaxi enriquecida com diferentes concentrações de pimenta.

\begin{tabular}{ccc}
\hline Tratamentos & CTO (NMP/g ) & CTT (NMP/g) \\
\hline T1 & $<3,0$ & $<3,0$ \\
T2 & $<3,0$ & $<3,0$ \\
T3 & $<3,0$ & $<3,0$ \\
T4 & $\leq 3,0$ & $<3,0$ \\
\hline
\end{tabular}

Fonte: Arquivo Pessoal (2018).

Os resultados obtidos nos testes para Coliformes Totais e de para Coliformes Termotolerantes, confirmam que as amostras 
apresentaram o NMP menor que 3, indicando que a geleia está livre de coliformes totais e coliformes termotolerantes e que a aplicação das boas práticas de fabricação foram efetivas para a manutenção da qualidade e da segurança do produto. Segundo Granada et al. (2005), foram observados resultados negativos por contaminação com Coliformes para geleia de abacaxi, desta maneira seguindo os padrões estabelecidos pela Secretaria de Vigilância Sanitária com Resolução RDC n 12 , de 02 de janeiro de 2001.

Não houve diferença estatística significativa entre os tratamentos da geleia de abacaxi enriquecida com pimenta para a variável pH, com uma média geral de 4,15 (Tabela 2). Isto demonstra que a adição de pimenta bode à geleia de abacaxi não promoveu alteração nos valores de pH. Estes resultados corroboram com os encontrados por Pereira et al., (2015), que encontraram valores de $\mathrm{pH}$ próximos de 3,94. Ferdous \& Alim (2018) obtiveram médias de 4,21 de pH em geleias de abacaxi, superiores mesmo sem a adição de pimenta. Valores de $\mathrm{pH}$, abaixo ou acima daqueles considerados ótimos para formação de gel (3,0 a 3,2), diminuem a firmeza do produto final (Fundação Centro Tecnológico de Minas Gerais, 1985).

Tabela 2. Resultados de $\mathrm{pH}$, acidez e umidade para geleia de abacaxi enriquecida com Pimenta.

\begin{tabular}{ccccc}
\hline Amostra & $\mathrm{pH}$ & Acidez & Umidade & Cinzas \\
\hline T1 & $4,01 \mathrm{a}$ & $0,32 \mathrm{~b}$ & $13,27 \mathrm{a}$ & $23,76 \mathrm{a}$ \\
$\mathrm{T} 2$ & $4,00 \mathrm{a}$ & $0,34 \mathrm{ab}$ & $8,15 \mathrm{~b}$ & $22.85 \mathrm{a}$ \\
$\mathrm{T} 3$ & $3,92 \mathrm{a}$ & $0,32 \mathrm{ab}$ & $12,56 \mathrm{ab}$ & $26,32 \mathrm{a}$ \\
$\mathrm{T} 4$ & $4,65 \mathrm{a}$ & $0,34 \mathrm{a}$ & $9,77 \mathrm{ab}$ & $25,18 \mathrm{a}$ \\
\hline Média Geral & $4,15 \pm 0,52$ & $0,33 \pm 0,01$ & $10,94 \pm 1,85$ & $24,53 \pm 2,11$ \\
\hline CV $(\%)$ & 12,66 & 2,56 & 16,94 & 8,61 \\
\hline
\end{tabular}

As médias seguidas pela mesma letra, não se diferem estatisticamente pelo teste de Tukey ao nível de 5\% de probabilidade. Fonte: Arquivo Pessoal (2018).

Para a acidez total titulável, os tratamentos T2 $(0,34)$ e T4 $(0,34)$, apresentaram média superior aos demais tratamentos. Os tratamentos T1 $(0,32)$ e T4 $(0,34)$ diferiram entre si. Carneiro et al., (2012), encontraram, em geleia de pêssego, valores de acidez entre 0,47 a 0,01 . Os resultados obtidos foram superiores aos encontrados por Pereira et al., (2015), que obtiveram 0,24 de acidez titulável em geleia de abacaxi tipo pérola, mas em contrapartida foram inferiores aos resultados obtidos por Silva et al. (2019), que encontrou valores de até 0,82 em geleia de abacaxi enriquecida com diferentes concentrações de hibisco. Dessa forma, pode-se notar a influência da adição de diferentes concentrações de pimenta bode, frente aos valores de acidez titulável da geleia. Esse incremento da acidez se deu pelo aumento do teor de ácidos orgânicos presentes na pimenta.

Para o teor de umidade, o tratamento $1(13,27 \%)$ apresentou média superior ao tratamento $2(8,15 \%)$, e os tratamentos 3 e 4 não apresentaram diferença significativa entre si. Entretanto, todos os tratamentos se enquadram no que determina a Resolução CNNPA 12 de 1978, que estabelece o máximo de 35\% de umidade em geleias de frutas do tipo extra (Brasil 1978). Segundo Teles et al. (2017), na fabricação de geleia de pimenta comum e extra com graviola, foram encontrados valores de umidade entre $28,72 \%$ a 37,45\%. Para o teor de cinzas, não houve diferença significativa entre os tratamentos. Uma forma de estimar a quantidade de minerais de uma amostra é fazendo uma análise de suas cinzas. Após a carbonização da matéria orgânica de alimento, a cinza residual corresponde à matéria inorgânica do mesmo. Teores de cinzas parecidos foram reportados por Silva et al., (2019) e também Ferdous e Alim (2018), na geleia de abacaxi. Nesta, pode-se encontrar vários minerais em diferentes quantidades, porém, alguns deles podem ser perdidos durante o processo, devido às altas temperaturas, como é o caso do manganês (Cecchi 2003). 
Não houve, diferença estatística quanto aos atributos de cor entre as amostras (Tabela 3). Para os demais atributos analisados como consistência, aroma, aspecto geral e sabor, a amostra T1 apresentou maior média, se diferenciando estatisticamente entre as demais amostras que apresentaram menor valor sendo semelhantes estatisticamente. As características de qualidade sensorial, tais como sabor, textura e aparência, precisam ser monitoradas desde o momento da percepção e escolha desta qualidade, por meio de estudos de consumidor que determine perfis de consumo (Lima et al., 2017).

Tabela 3. Resultados da análise sensorial da geleia de abacaxi enriquecida com pimenta.

\begin{tabular}{ccccccc}
\hline Amostra & Consistência & Aroma & Cor & Aspecto Geral & Sabor & IA (\%) \\
\hline T1 & $8,04 \mathrm{a}$ & $7,98 \mathrm{a}$ & $7,80 \mathrm{a}$ & $8,16 \mathrm{a}$ & $8,64 \mathrm{a}$ & $90,26 \mathrm{a}$ \\
T2 & $6,78 \mathrm{~b}$ & $7,32 \mathrm{~b}$ & $7,34 \mathrm{a}$ & $7,18 \mathrm{~b}$ & $7,46 \mathrm{~b}$ & $80,17 \mathrm{~b}$ \\
T3 & $7,30 \mathrm{~b}$ & $7,14 \mathrm{~b}$ & $7,46 \mathrm{a}$ & $7,38 \mathrm{~b}$ & $7,30 \mathrm{~b}$ & $81,28 \mathrm{~b}$ \\
T4 & $7,24 \mathrm{~b}$ & $7,24 \mathrm{~b}$ & $7,72 \mathrm{a}$ & $7,36 \mathrm{~b}$ & $7,34 \mathrm{~b}$ & $82,00 \mathrm{~b}$ \\
\hline Média & $7,34 \pm 1,17$ & $7,42 \pm 1,14$ & $7,58 \pm 1,24$ & $7,52 \pm 1,05$ & $7,69 \pm 1,03$ & $83,43 \pm 2,62$ \\
\hline CV $(\%)$ & 15,99 & 15,37 & 16,42 & 14,01 & 13,39 & 3,14 \\
\hline
\end{tabular}

As médias seguidas pela mesma letra, não se diferem estatisticamente entre si pelo teste de Tukey ao nível de 5\% de probabilidade. Fonte: Arquivo Pessoal (2018).

Apenas o tratamento T1 se diferenciou estatisticamente dos demais tratamentos, enquanto os tratamentos T2, T3 e T4 não diferiram entre. Isto pode ser atribuído à dificuldade dos provadores de perceberem diferenças sutis nas concentrações de pimentas nas geleias devido à pungência. De maneira geral, os resultados demonstram que todas as geleias foram aceitas apresentando o índice de aceitabilidade superior a 70\%, porém a geleia que apresentou maior aceitabilidade foi a do tratamento $1(90,26 \%)$ com 0,2 gramas de pimenta apresentando a menor quantidade de pimenta e pungência. Araújo et al. (2012) trabalhando com geleia de pimenta com abacaxi, verificaram que a amostra com maior quantidade de pimenta, porém com menor teor de pungência, foi a mais aceita diante da análise sensorial. Araújo et al. (2014) também trabalhando com geleia de pimenta com acerola, verificaram que a melhor aceitação da amostra que apresentava em sua composição maior quantidade de pimenta com menor teor de pungência. Estes resultados demonstram uma preferência maior dos provadores por geleias com menores concentrações de pimenta.

\section{Considerações Finais}

De acordo com os resultados da análise microbiológica da geleia de abacaxi enriquecida com pimenta, o produto estava dentro dos padrões legais estabelecidos, estando aptas para o consumo. A geleia com menor concentração de pimenta (T1) obteve maior aceitação sensorial (90,26\%) para todos os atributos avaliados, porém, todas as amostras foram bem aceitas pelos consumidores, apresentando índice de aceitabilidade superior a 70\%, que é o mínimo para um produto ser aceito. Para o valor de acidez total titulável, o tratamento T4 apresentou-se mais ácido que os demais tratamentos. Diante dos resultados obtidos, recomenda-se a formulação do tratamento T1 por ter maior aceitação dos provadores e por atender aos padrões de controle de qualidade da legislação brasileira.

\section{Referências}

Araújo, E. R., Silva, P. K., do Nascimento, M. F., Nascimento, N. F. F., Amorim Barral, M. A., Rêgo, M. M., \& Rêgo, E. R. (2014). Desenvolvimento de geleia de pimenta com acerola: Análise sensorial e aceitação comercial. Revista AGROTEC Agropecuária Técnica, 35(1), 81-88. 
Research, Society and Development, v. 10, n. 8, e54310817718, 2021

(CC BY 4.0) | ISSN 2525-3409 | DOI: http://dx.doi.org/10.33448/rsd-v10i8.17718

Araújo, E. R., Rêgo, E. R., Sapucay, M. J. L. C., Rêgo, M. M. \& Santos, R. M. C. (2012). Elaboração e análise sensorial de geleia de pimenta com abacaxi. Revista Brasileira de Produtos Agroindustriais, 14(1), 233-238.

Barduzzi, J. F. (2011). Extração e Quantificação da Capsaicina em Pimenta Dedo-de-Moça. Fundação Educacional do Município de Assis - FEMA

Brasil, Ministério da Saúde. (1978). Agência Nacional de Vigilância Sanitária fixa os padrões de identidade e qualidade para os alimentos (e bebidas). Resolução CNNPA n. 12, de 24 de setembro de (1978). Diário Oficial da União.

Carneiro, A. P. G., Costa, E. A., Soares, D. J., Moura, S. M. \& Constant, P. B. L. (2012). Caracterização físico-química dos frutos in natura e geleias de morango e pêssego, e aspectos de rotulagem do produto ao consumidor. Revista Brasileira de Produtos Agroindustriais, [S.L.], 14(3), 295-298.

Cecchi, H. M. (2000). Fundamentos teóricos e práticos em análise de alimentos. (2a ed.), Editora da Unicamp.

Cruz, D. R. C., Virtuoso, M. C. S., Faria, L. R., Cabral, F. S., Sarti, J. K. \& Vale, L. S. R. (2019). Avaliação morfológica de genótipos de pimentas bode desenvolvidos no IF Goiano - Campus Ceres. Revista Brasileira Multidisciplinar, 22(3), 2-10.

Ferdous, M. J. \& Alim, M. A. (2018). Physico-chemical properties of mixed jam from pineapple and sweet gourd. Journal of the Bangladesh Agricultural University, 16(2), 309-314.

Ferreira, D. F. (2014). Sisvar. Versão 5.6. UFLA/DEX.

Fundação Centro Tecnológico De Minas Gerais. (1985). Manual para fabricação de geléias. Belo Horizonte: Governo do Estado de Minas Gerais/Secretaria do Estado de Ciência e Tecnologia.

Granada, G. G., Zambiazi, R. C., Mendonça, C. R. B. \& Silva, E. (2005). Caracterização física, química, microbiológica e sensorial de geléias light de abacaxi. Ciência e Tecnologia de Alimentos, 25(4), 629-635.

Instituto Adolfo Lutz. (2008). Métodos físico-químicos para análise de alimentos /coordenadores Odair Zenebon, Neus Sadocco Pascuet e Paulo Tiglea.: Instituto Adolfo Lutz, p. 1020

Lima, P. C., Souza, B. S., Santini, A. T. \& Oliveira, D. C. (2017). Aproveitamento agroindustrial de resíduos provenientes do abacaxi 'PÉROLA' minimamente processado. Holos, 2(1),122-132.

Macedo, A. P., Bonatto, E. C. S., Sousa, A. F., Lamarão, C. V. \& Saraiva, M. G. G. (2017). Desenvolvimento e aceitabilidade de geleias com pimentas murupi (Capsicum Chinese Jaq.) e malagueta (Capsicum Frutescens L.). Universidade Federal do Amazonas, Manaus-AM.

Melo, C. M. T., Costa, L. A., Bonnas, D. S. \& Chang R. (2011). Compostos Fenólicos e Capacidade Antioxidante de Pimentas Capsicum chinense (bode), Capsicum baccatum variedade praetermissum (cumari) e Capsicum frutescens (malagueta). Instituto Federal do Triângulo Mineiro-Campus Uberlândia.

Minim, V. P. R. (2010). Análise sensorial: estudos com consumidores. Ed. UFV. 308p.

Nascimento, W. M., Dias, D. C. F. S. \& Freitas, R. A. (2006). Produção de sementes de pimenta. Informe Agropecuário: Cultivo da pimenta, Belo Horizonte, 30-39.

Pereira, E. M., Leite Filho, M. T., Santos, Y. M. G., Pereira, B. B. M. \& Maracajá, P. B. (2015). Elaboração e qualidade de geleia e composta de abacaxi "pérola". Revista Verde de Agroecologia e Desenvolvimento Sustentável, 10(2), 149-153.

Raghuwanshi, S., Kumar, T., Sah, P. K., Pahade, D., Samskruthi, G., Prakash, V. U. B. \& Lavanya, K. (2019). Physicochemical and organoleptic properties of spray-dried pineapple powder: Effect of maltodextrin concentration and inlet air temperature. International Journal of Chemical Stuies, 7(3), $1030-1034$.

Rodrigues, I. C., Asquieri, E. R., Silva, A. G. M., Damiani, C. (2020). Estudo do processamento de geleia de melancia enriquecida com extratos de jabuticaba e extrato de sementes de chia: características físico-químicas e potencial antioxidante. Research, Society And Development, 9(5), e45952934.

Shaari, N. A., Sulaiman, R., Rahman, R. A. \& Bakar, J. (2018). Production of pineapple fruit (Ananas comosus) powder using foam mat drying: effect of whipping time and egg albumen concentration. Journal of Food Processing and Preservation, 42(2), 1-13.

Shinwari, K. J \& Rao, P. S. (2018). Stability of bioactive compounds in fruit jam and jelly during processing and storage: A review. Trends in food science \& technology, 75(1), 181-193.

Silva, E. T. V., Moura, H. V., Figueiredo, R. M. F., Queiroz, A. J. M., Moreira, I. S. (2019). Production and characterization of mixed pineapple and hibiscus jam. Revista Agro@Mbiente On-Line, 13(1), 155-163.

Silva, N., Junqueira, V. C. A., Silveira, N. F. A. (1997). Manual de métodos de análise microbiológica de alimentos. Varela. 295p.

Souza, T. (2017). Instrutor ensina a fazer geleia de abacaxi com pimenta, veja receita. https://g1.globo.com/go/goias/noticia/instrutor-ensina-a- fazer-geleiade-abacaxi-com-pimenta-veja-receita.html

Teles, A. C. M., Pinto, E. G., Santos, J. R., Oliveira, C. F. D. \& Soares, D. S. B. (2017). Desenvolvimento e caracterização físico-química de geleia comum e extra de graviola com pimenta. Revista de Agricultura Neotropical, 4(1), 72-77. 